\title{
Thermodynamics of Quantum Information Flows
}

\author{
Krzysztof Ptaszyński ${ }^{1, *}$ and Massimiliano Esposito ${ }^{2}$ \\ ${ }^{1}$ Institute of Molecular Physics, Polish Academy of Sciences, Mariana Smoluchowskiego 17, 60-179 Poznań, Poland \\ ${ }^{2}$ Complex Systems and Statistical Mechanics, Physics and Materials Science Research Unit, University of Luxembourg, \\ L-1511 Luxembourg, Luxembourg
}

(Received 7 January 2019; revised manuscript received 4 March 2019; published 16 April 2019)

\begin{abstract}
We report two results complementing the second law of thermodynamics for Markovian open quantum systems coupled to multiple reservoirs with different temperatures and chemical potentials. First, we derive a nonequilibrium free energy inequality providing an upper bound for a maximum power output, which for systems with inhomogeneous temperature is not equivalent to the Clausius inequality. Second, we derive local Clausius and free energy inequalities for subsystems of a composite system. These inequalities differ from the total system one by the presence of an information-related contribution and build the ground for thermodynamics of quantum information processing. Our theory is used to study an autonomous Maxwell demon.
\end{abstract}

DOI: 10.1103/PhysRevLett.122.150603

The second law of thermodynamics is one of the main principles of physics. Within equilibrium thermodynamics there exist two equivalent formulations of this law. The first, referred to as the Clausius inequality, states that the sum of the entropy change of the system $\Delta S$ and the entropy exchanged with the environment $\Delta S_{\text {env }}$ during the transition between two equilibrium states is non-negative: $\Delta S+\Delta S_{\text {env }} \geq 0$. The exchanged entropy can be further expressed as $\Delta S_{\text {env }}=-Q / T$, where $Q$ is the heat delivered to the system. An alternative formulation, referred to as the free energy inequality, states that during the transition between two equilibrium states $W-\Delta F \geq 0$, where $W$ is the work performed on the system and $F=E-T S$ is the free energy (here $E$ denotes the internal energy). The latter formulation can be obtained from the former by using the first law of thermodynamics, $\Delta E=W+Q$.

Whereas these standard definitions of the second law apply when considering transitions between equilibrium states, the last few decades have brought significant progress towards generalizing them to both classical [1-7] and quantum [8-10] systems far from equilibrium. The most common formulation generalizes the Clausius inequality by stating that the average entropy production $\sigma$ is non-negative. For a large class of systems [4-6,9] the entropy production can be defined as $\sigma \equiv \Delta S-\sum_{\alpha} Q_{\alpha} \beta_{\alpha}$, where $\Delta S$ is the change of the Shannon or the von Neumann entropy of the system (which is well defined also out of equilibrium) and $Q_{\alpha}$ is the heat delivered to the system from the reservoir $\alpha$ with the inverse temperature $\beta_{\alpha}$; additionally, in Markovian systems the entropy production rate $\dot{\sigma}$ is always non-negative $[7,10]$. Formulations generalizing the free energy inequality [11-14] are much less common and have been so far confined mainly to systems coupled to an environment with a homogeneous temperature; for an exception, see Ref. [14].
These developments have also brought a deeper understanding of the relation between thermodynamics and the information theory $[12,15]$. One of the most important achievements is related to the field of thermodynamics of feedback-controlled systems [16]. Following the groundbreaking ideas of the Maxwell demon [17] and the Szilard engine [18], it was verified both theoretically [19-23] and experimentally [24-29] that by employing feedback one can reduce entropy of the system without exchanging heat. In such a case, modified Clausius inequalities, which relate the entropy change to the information flow, have to be applied [11-14,30-42]. It was also realized that the feedback control does not require the presence of any intelligent being (as in the original idea of Maxwell) but may be performed by an autonomous stochastic system coupled to the controlled one [43]. A consistent mathematical description of thermodynamics of autonomous information flow has been, however, so far confined mainly to classical stochastic systems with a special topology of network of jump processes, referred to as bipartite [38,39] or, in general, multipartite [41] systems.

Our work adds two new contributions to the field. First, we generalize the free energy inequality to Markovian open quantum systems coupled to reservoirs with different temperatures and show that this formulation of the second law is, in general, not equivalent to the Clausius inequality. Second, we formulate a consistent thermodynamic formalism describing thermodynamics of continuous information flow in a generic composite open quantum system and demonstrate the relation between the information and the nonequilibrium free energy. The applicability of our results is demonstrated on a quantum autonomous Maxwell demon based on quantum dots.

Nonequilibrium Clausius inequality.-We consider a generic open quantum system weakly coupled to $N$ 
equilibrated reservoirs $\alpha$ with temperatures $T_{\alpha}$ (inverse temperatures $\beta_{\alpha} \equiv 1 / T_{\alpha}$ ) and chemical potentials $\mu_{\alpha}$, described by the time-independent Hamiltonian

$$
\hat{H}=\hat{H}_{S}+\hat{H}_{B}+\hat{H}_{I},
$$

where $\hat{H}_{S}, \hat{H}_{B}, \hat{H}_{I}$ are, correspondingly, the Hamiltonian of the system, reservoirs, and interaction of the system with the reservoirs. Within the Markov approximation the (reduced) density matrix of the system evolves according to the master equation [44]

$$
d_{t} \rho=-i\left[\hat{H}_{\mathrm{eff}}, \rho\right]+\mathcal{D} \rho,
$$

where $\rho$ is the density matrix, $d_{t}$ denotes the total derivative of the function, $\hat{H}_{\text {eff }}$ is the effective Hamiltonian of the system (it may differ from $\hat{H}_{S}$ due to coupling to the environment [44]), and $\mathcal{D}$ is the superoperator describing the dissipative dynamics. Here and from here on we take $\hbar=k_{B}=1$. We further assume that the dissipator $\mathcal{D}$ is of Lindblad form, thus ensuring a completely positive tracepreserving dynamics [45,46], and that $\hat{H}_{\text {eff }}$ commutes with $\hat{H}_{S}$, which is justified by the perturbation theory (see the Supplemental Material [47]). Furthermore, within the Markov approximation the dissipation is additive; i.e., the superoperator $\mathcal{D}$ can be represented as a sum of dissipators associated with each reservoir, denoted as $\mathcal{D}^{\alpha}: \mathcal{D}=\sum_{\alpha} \mathcal{D}^{\alpha}[10,54]$. For violation of additivity beyond the weak coupling regime, see Refs. [57-60].

We also assume that the grand canonical equilibrium state (Gibbs state) with respect to the reservoir $\alpha$,

$$
\rho_{\mathrm{eq}}^{\alpha}=Z_{\beta_{\alpha}, \mu_{\alpha}}^{-1} e^{-\beta_{\alpha}\left(\hat{H}_{S}-\mu_{\alpha} \hat{N}\right)},
$$

is a stationary state of $\mathcal{D}^{\alpha}$, i.e., $\mathcal{D}^{\alpha} \rho_{\text {eq }}^{\alpha}=0$ [10]; here $Z_{\beta_{\alpha}, \mu_{\alpha}}=\operatorname{Tr}\left\{\exp \left[-\beta_{\alpha}\left(\hat{H}_{S}-\mu_{\alpha} \hat{N}\right)\right]\right\}$ is the partition function and $\hat{N}$ is the particle number operator. This assumption guarantees that for an arbitrary form of dissipator the Gibbs state is a stationary state at equilibrium (i.e., for equal temperatures and chemical potentials of the reservoirs), which is true for systems weakly coupled to the environment [44]. Let us then apply Spohn's inequality [8],

$$
-\operatorname{Tr}\left[\left(\mathcal{D}^{\alpha} \rho\right)\left(\ln \rho-\ln \rho_{\mathrm{eq}}^{\alpha}\right)\right] \geq 0,
$$

which is valid for any superoperator $\mathcal{D}^{\alpha}$ of Lindblad form with a steady state $\rho_{\text {eq }}^{\alpha}$ (not necessarily a unique steady state). As a result, one obtains the partial Clausius inequality for entropy production associated with each dissipator [10],

$$
\dot{\sigma}_{\alpha}=\dot{S}^{\alpha}-\beta_{\alpha} \dot{Q}_{\alpha} \geq 0
$$

where

$$
\dot{S}^{\alpha}=-\operatorname{Tr}\left[\left(\mathcal{D}^{\alpha} \rho\right) \ln \rho\right]
$$

is the rate of change of the von Neumann entropy of the system $S=-\operatorname{Tr}(\rho \ln \rho)$ due to the dissipator $\mathcal{D}^{\alpha}$, and

$$
\dot{Q}_{\alpha}=\operatorname{Tr}\left[\left(\mathcal{D}^{\alpha} \rho\right)\left(\hat{H}_{S}-\mu_{\alpha} \hat{N}\right)\right]
$$

is the heat current from the reservoir $\alpha$. Summing all the rates $\dot{S}^{\alpha}$, one gets the total derivative of the von Neumann entropy: $d_{t} S=\sum_{\alpha} \dot{S}^{\alpha}$. Therefore, summing up Eq. (5) over the reservoirs $\alpha$, one recovers the standard Clausius inequality [10]

$$
\dot{\sigma} \equiv \sum_{\alpha} \dot{\sigma}_{\alpha}=d_{t} S-\sum_{\alpha} \beta_{\alpha} \dot{Q}_{\alpha} \geq 0
$$

where $\dot{\sigma}$ is the total entropy production rate. We note that the rates $\dot{S}^{\alpha}$ can be nonzero also at the steady state, when $d_{t} S=0$ and the total entropy production is fully determined by the heat flows.

Nonequilibrium free energy inequality.-Let us now define energy and work currents to the lead $\alpha$ as

$$
\begin{gathered}
\dot{E}_{\alpha}=\operatorname{Tr}\left[\left(\mathcal{D}^{\alpha} \rho\right) \hat{H}_{S}\right], \\
\dot{W}_{\alpha}=\mu_{\alpha} \operatorname{Tr}\left[\left(\mathcal{D}^{\alpha} \rho\right) \hat{N}\right],
\end{gathered}
$$

such that $\dot{E}_{\alpha}=\dot{Q}_{\alpha}+\dot{W}_{\alpha}$ and $\sum_{\alpha} \dot{E}_{\alpha}=d_{t} E$, where $E=$ $\operatorname{Tr}\left(\rho \hat{H}_{S}\right)$ is the internal energy. Since we assume the Hamiltonian to be time independent, we consider only chemical and not mechanical work. Multiplying Eq. (5) by $T_{\alpha}$ and replacing $\dot{Q}_{\alpha} \rightarrow \dot{E}_{\alpha}-\dot{W}_{\alpha}$, one gets

$$
T_{\alpha} \dot{\sigma}_{\alpha}=\dot{W}_{\alpha}-\dot{\mathcal{F}}_{\alpha} \geq 0,
$$

where $\dot{\mathcal{F}}_{\alpha} \equiv \dot{E}_{\alpha}-T_{\alpha} \dot{S}^{\alpha}$ is the partial nonequilibrium free energy rate. Summing over $\alpha$ one obtains the nonequilibrium free energy inequality

$$
\sum_{\alpha} T_{\alpha} \dot{\sigma}_{\alpha}=\dot{W}-\dot{\mathcal{F}} \geq 0,
$$

where $\dot{W} \equiv \sum_{\alpha} \dot{W}_{\alpha}$ is the total work rate, and

$$
\dot{\mathcal{F}} \equiv \sum_{\alpha} \dot{\mathcal{F}}_{\alpha}=d_{t} E-\sum_{\alpha} T_{\alpha} \dot{S}^{\alpha}
$$

is the total nonequilibrium free energy rate. Equation (12) is a complementary formulation of the second law of thermodynamics. From a practical point of view, it provides an upper bound for the maximum work output. At the steady state $d_{t} E=0$, and thus the system can perform work $(\dot{W}<0)$ only when a temperature difference between the reservoirs is present. 
Let us emphasize that Eqs. (8) and (12) are, in general, not equivalent; the former corresponds to the sum of partial Clausius inequalities [Eq. (5)], whereas the latter corresponds to the weighted sum, in which Eq. (5) is multiplied by a local temperature $T_{\alpha}$. They become equivalent only when the system is attached to an isothermal environment, i.e., $T_{\alpha}=T$. Then the rate $\dot{\mathcal{F}}$ can be identified as the total derivative of the state function $F: \dot{\mathcal{F}}=d_{t} F=d_{t}(E-T S)$. At the steady state $d_{t} F=0$, and thus $\dot{W}>0$. This corresponds to the Kelvin-Planck statement of the second law, according to which one cannot continuously generate work by cooling an isothermal environment.

Local Clausius inequality.-Let us now consider a system made of two coupled subsystems described by the Hamiltonian

$$
\hat{H}_{S}=\hat{H}_{1}+\hat{H}_{2}+\hat{H}_{12},
$$

where $\hat{H}_{i}$ is the Hamiltonian of the subsystem $i=1,2$ and $\hat{H}_{12}$ is the interaction Hamiltonian. We also assume that each subsystem is attached to a separate set of reservoirs; baths coupled to the subsystem $i$ will be then denoted as $\alpha_{i}$. By summing Eq. (5) over the reservoirs $\alpha_{i}$, one obtains

$$
\dot{\sigma}_{i} \equiv \sum_{\alpha_{i}} \dot{\sigma}_{\alpha_{i}}=\sum_{\alpha_{i}} \dot{S}^{\alpha_{i}}-\sum_{\alpha_{i}} \beta_{\alpha_{i}} \dot{Q}_{\alpha_{i}} \geq 0 .
$$

Here $\dot{\sigma}_{i}=\sum_{\alpha_{i}} \dot{\sigma}_{\alpha_{i}}$ denotes the local entropy production; it is an extensive quantity, i.e., $\dot{\sigma}=\dot{\sigma}_{1}+\dot{\sigma}_{2}$. We will now transform Eq. (15) to a form illustrating the relation between entropy and information. Let us remind the reader that the quantum mutual information is defined as $I_{12}=S_{1}+S_{2}-S$, where $S_{i}=-\operatorname{Tr}\left(\rho_{i} \ln \rho_{i}\right)$ is the von Neumann entropy of the subsystem $i$ [55] (here $\rho_{i}$ is the reduced density matrix of the subsystem $i$ ). We can then separate the total derivative of the mutual information into two contributions, $d_{t} I_{12}=\dot{I}_{1}+\dot{I}_{2}$, where

$$
\dot{I}_{i} \equiv d_{t} S_{i}-\sum_{\alpha_{i}} \dot{S}^{\alpha_{i}}
$$

Here we have applied the identity $\sum_{\alpha} \dot{S}^{\alpha}=d_{t} S$. The rate $\dot{I}_{i}$ can be calculated as

$$
\dot{I}_{i}=-\operatorname{Tr}\left(d_{t} \rho_{i} \ln \rho_{i}\right)+\operatorname{Tr}\left[\left(\mathcal{D}_{i} \rho\right) \ln \rho\right],
$$

where $\mathcal{D}_{i}=\sum_{\alpha_{i}} \mathcal{D}^{\alpha_{i}}$ is the dissipator associated with the subsystem $i$. The rate $\dot{I}_{i}$ can be further decomposed into contributions related to the unitary and the dissipative dynamics; see the Supplemental Material [47] for details.

Replacing $\sum_{\alpha_{i}} \dot{S}^{\alpha_{i}} \rightarrow d_{t} S_{i}-\dot{I}_{i}$ in Eq. (15), one obtains the local Clausius inequlity relating the entropy balance of the subsystem $i$ to the information flow:

$$
\dot{\sigma}_{i}=d_{t} S_{i}-\sum_{\alpha_{i}} \beta_{\alpha_{i}} \dot{Q}_{\alpha_{i}}-\dot{I}_{i} \geq 0 .
$$

This inequality is identical in form to the one previously derived in Ref. [39]. However, our result is much more general. First, it enables one to describe systems undergoing a quantum dynamics formulated in terms of a density matrix, whereas the former approach was purely classical and formulated in terms of probabilities. Second, our result has a much wider range of applicability even in the classical limit. Indeed, the approach from Ref. [39] was restricted to so-called bipartite systems, which exclude stochastic transitions generating a simultaneous change of states of both subsystems. However, two-component open quantum systems are, in general, not bipartite, even when their populations obey a classical master equation. Instead, our only requirement is that the dissipation is additive; i.e., one can split the dissipator $\mathcal{D}$ into contributions $\mathcal{D}_{i}$ in a physically meaningful way. The system can become bipartite when the total Hamiltonian $\hat{H}_{S}$ commutes with the subsystem Hamiltonian $\hat{H}_{i}$ and one applies the effectively classical description by means of the secular (rotating wave) approximation; in such a case our approach reduces to that from Ref. [39]. We discuss these issues is detail in the Supplemental Material [47].

Let us finally emphasize that all of the previous discussion can be easily generalized to the multicomponent systems consisting of $M$ subsystems. Then $\sum_{i} \dot{I}_{i}=d_{t} I_{1, \ldots, M}$, where $I_{1, \ldots, M} \equiv \sum_{i} S_{i}-S$ is the multipartite mutual information [61].

Local free energy inequality.-Analogously, summing up Eq. (11) over reservoirs $\alpha_{i}$, we derive an inequality describing the nonequilibrium free energy balance for a single subsystem:

$$
\sum_{\alpha_{i}} T_{\alpha_{i}} \dot{\sigma}_{\alpha_{i}}=\dot{W}_{i}-\dot{\mathcal{F}}_{i} \geq 0,
$$

where $\dot{W}_{i} \equiv \sum_{\alpha_{i}} \dot{W}_{\alpha_{i}}$ and $\dot{\mathcal{F}}_{i} \equiv \sum_{\alpha_{i}} \dot{\mathcal{F}}_{\alpha_{i}}$.

As in the case of the total system, Eqs. (18) and (19) are nonequivalent and complementary formulations of the local second law of thermodynamics. They become equivalent when the subsystem $i$ is coupled to an isothermal environment with a single temperature $T_{i}$. Then $T_{i} \dot{I}_{i}=$ $-T_{i} \sum_{\alpha_{i}} \dot{S}^{\alpha_{i}}$, and therefore

$$
\dot{\mathcal{F}}_{i}=\dot{E}_{i}+T_{i} \dot{I}_{i}
$$

The local nonequilibrium free energy rate consists therefore of the energy-related and the information-related contribution. At the steady state the internal energy of the system is constant $\left(d_{t} E=0\right)$, and thus $E_{i}$ can be interpreted as the energy flow to the subsystem $j \neq i$. The subsystem attached to an isothermal environment may therefore 


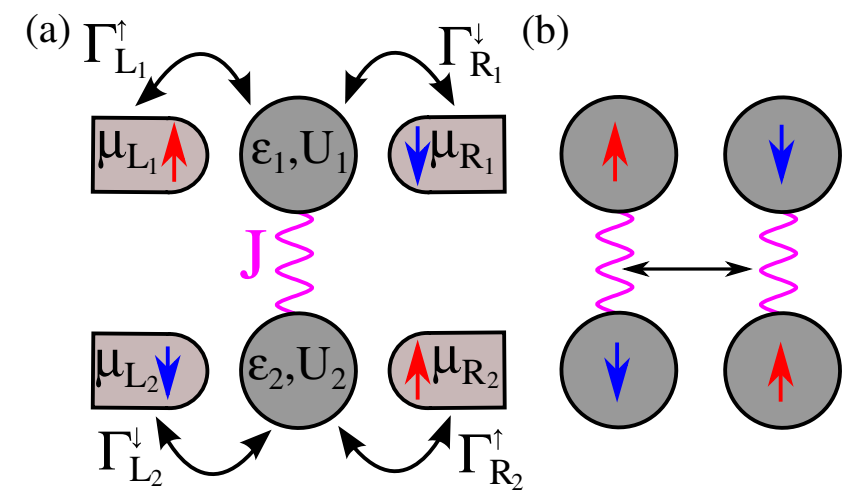

FIG. 1. (a) Scheme of the autonomous quantum Maxwell demon described in the text. (b) Schematic representation of the spin exchange induced by the $X Y$ interaction.

perform work either due to the energy flow from the other subsystem or due to the information flow; the latter case corresponds to the operation of information-powered devices.

Example.-The applicability of our approach will now be demonstrated on a recently proposed [62] model of an autonomous quantum Maxwell demon. Here we describe the device only briefly; for more details we refer the reader to the original paper.

The analyzed setup [Fig. 1(a)] is composed of two quantum dots coupled by the $X Y$ exchange interaction, each attached to two electrodes with equal temperatures $T$. The Hamiltonian of the system is defined as

$$
\begin{aligned}
\hat{H}_{S}= & \sum_{i \sigma} \epsilon_{i} d_{i \sigma}^{\dagger} d_{i \sigma}+\sum_{i} U_{i} n_{i \uparrow} n_{i \downarrow} \\
& +\frac{J}{2}\left(d_{1 \uparrow}^{\dagger} d_{1 \downarrow} d_{2 \downarrow}^{\dagger} d_{2 \uparrow}+d_{1 \downarrow}^{\dagger} d_{1 \uparrow} d_{2 \uparrow}^{\dagger} d_{2 \downarrow}\right),
\end{aligned}
$$

where $d_{i \sigma}^{\dagger}\left(d_{i \sigma}\right)$ is the creation (annihilation) operator of an electron with spin $\sigma \in\{\uparrow, \downarrow\}$ in the dot $i \in\{1,2\}, n_{i \sigma}=$ $d_{i \sigma}^{\dagger} d_{i \sigma}$ is the particle number operator, $\epsilon_{i}$ is the orbital energy, $U_{i}$ is the intradot Coulomb interaction in the dot $i$, and $J$ is the exchange coupling. The bath Hamiltonian reads $\hat{H}_{B}=\sum_{\alpha_{i} k \sigma} \epsilon_{\alpha_{i} k \sigma} c_{\alpha_{i} k \sigma}^{\dagger} c_{\alpha_{i} k \sigma}$, where $c_{\alpha_{i} k \sigma}^{\dagger}\left(c_{\alpha_{i} k \sigma}^{\dagger}\right)$ is the creation (annihilation) operator of an electron with spin $\sigma$, wave number $k$, and energy $\epsilon_{\alpha_{i} k \sigma}$ in the reservoir $\alpha_{i}$; here $\alpha_{i}=L_{i}$ $\left(R_{i}\right)$ denotes the left (right) reservoir attached to the dot $i$. Finally, the system bath-interaction Hamiltonian is expressed as $\hat{H}_{I}=\sum_{i \alpha_{i} k \sigma} t_{\alpha_{i}} c_{\alpha_{i} k \sigma}^{\dagger} d_{i \sigma}+$ H.c., where $t_{\alpha_{i}}$ is the tunnel coupling of the dot $i$ to the reservoir $\alpha_{i}$. We also define the coupling strength $\Gamma_{\alpha_{i}}^{\sigma}=2 \pi\left|t_{\alpha_{i}}\right|^{2} \rho_{\alpha_{i}}^{\sigma}$, where $\rho_{\alpha_{i}}^{\sigma}$ is the density of states of electrons with spin $\sigma$ in the bath $\alpha_{i}$.

To describe the dynamics of the device, we apply a microscopically derived Lindblad equation which couples populations to coherences and is thermodynamically consistent in the weak coupling limit (it is equivalent to the phenomenological approach proposed in Ref. [56]). Details of the method, a discussion of its limits of validity, and a comparison with the secular Lindblad equation (which is by construction thermodynamically consistent but neglects genuine quantum coherent effects) are presented in the Supplemental Material [47].

The device works as follows: The baths are taken to be fully spin polarized; i.e., either $\Gamma_{\alpha_{i}}^{\uparrow}$ or $\Gamma_{\alpha_{i}}^{\downarrow}$ is equal to 0 . As a result, the electrodes act as spin filters which forbid the tunneling of electrons with a spin opposite to the polarization $[63,64]$. Additionally, the polarizations of reservoirs attached to a single dot are arranged in an antiparallel way such that the electron may be transferred between the electrodes only when it changes its spin. This is enabled by the $X Y$ interaction which exchanges spins between the dots [Fig. 1(b)]. Since this interaction conserves the total spin, the spin flips occur simultaneously in both subsystems, and thus the steady-state currents through both dots have to be equal. Let us now apply a high positive bias $V_{1}=$ $\mu_{L_{1}}-\mu_{R_{1}}>0$ to the first dot, and a smaller opposite bias $V_{2}=\mu_{L_{2}}-\mu_{R_{2}}<0\left(\left|V_{2}\right|<\left|V_{1}\right|\right)$ to the other one. Then the voltage-driven current through the first dot will pump electrons in the second dot against the bias, which is due to a nonequilibrium spin population induced by spin flips. This can be interpreted as the operation of a Maxwell demon: The high positive voltage in the first dot tends to reset the upper dot to the state $\uparrow$ (i.e., the singly occupied state with a spin up). As a result, the spin dynamics generated by the $X Y$ interaction (equivalent to the operation of the quantum $i$ swAP gate [65]) flips the spin in the second dot if it is in the state $\downarrow$, and leaves it unchanged when it is in the state $\uparrow$ [cf. Fig. 1(b)], thus creating an excess population of spins $\uparrow$. This feedback mechanism induces the information flow between the dots, thus enabling a conversion of heat into work.

Our local Clausius and free energy inequalities [Eqs. (18) and (19)] for this system are demonstrated in Fig. 2. As one can see, for $J \lesssim 500 \Gamma$ the dot 2 cools its isothermal environment, which is enabled by the information flow $\left(T \dot{I}_{2}<-\dot{Q}_{2}<0\right)$; at the same time it performs work (i.e., pumps the current against the voltage), which is possible due to the negative free energy rate $\left(\dot{\mathcal{F}}_{2}<\dot{W}_{2}<0\right)$. This is compensated for by the dissipation of work into heat in the first dot. We emphasize that for $J \lesssim 50 \Gamma$ the work is performed in the second dot only due to feedback-induced information flow $\left(\dot{\mathcal{F}}_{2} \approx T \dot{I}_{2}\right)$ and not due to energy flow, which is negligible $\left(E_{2} \approx 0\right)$; this justifies the interpretation of the device as a Maxwell demon. As shown in the Supplemental Material [47], in this regime the information flow is generated by the unitary spin dynamics rather than the dissipative tunneling dynamics, in contrast to the classical Maxwell demon studied in Ref. [43]. On the other hand, for $J \gtrsim 50 \Gamma$ one can observe a noticeable energy flow from the first to the second dot which results from the splitting of energy levels. As a 


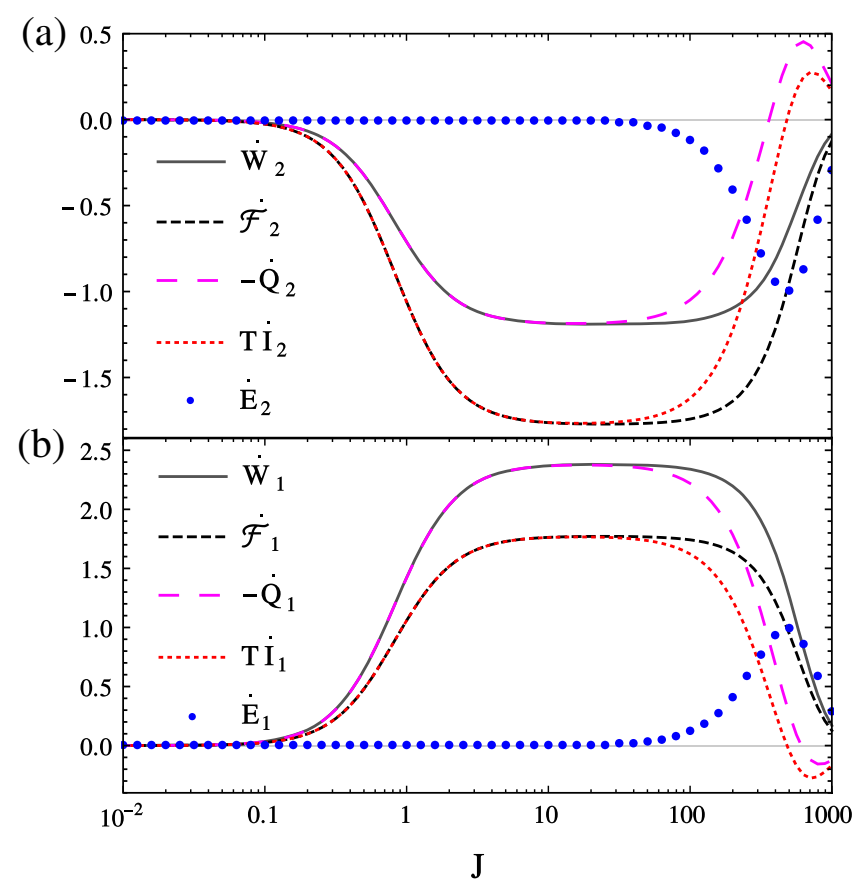

FIG. 2. Steady-state work, free energy, heat, information flow, and energy flow for (a) the second and (b) the first quantum dot. Results for $T=100, \epsilon_{i}=0, U_{i} \rightarrow \infty$ (strong Coulomb blockade), $\mu_{L_{1}}=-\mu_{R_{1}}=60, \mu_{L_{2}}=-\mu_{R_{2}}=-30, \Gamma_{L_{1}}^{\downarrow}=\Gamma_{R_{1}}^{\uparrow}=\Gamma_{L_{1}}^{\uparrow}=$ $\Gamma_{R_{2}}^{\downarrow}=0$, and all other coupling strengths $\Gamma_{\alpha_{i}}^{\sigma}$ equal to $\Gamma=1$.

consequence, for $J \gtrsim 500 \Gamma$ the $\operatorname{dot} 2$ starts to heat its environment $\left(-\dot{Q}_{2}>0\right)$, and the setup ceases to work as a Maxwell demon. However, the dot 2 still performs work thanks to the negativity of the nonequilibrium free energy rate $\dot{\mathcal{F}}_{2}$, which now includes a significant energy-related contribution $\dot{E}_{2}$.

Conclusions.-Our inequality (12), by providing a complement to the second law, may have novel implications for the design of quantum heat engines [66] which need to be explored. In turn, our inequalities (18) and (19) provide the basis for thermodynamics of quantum information processing. We hope that, as has happened with the classical counterpart of Eq. (18), numerous applications and experiments [25] will also ensure it in the quantum realm.

K. P. is supported by the National Science Centre, Poland, under the project Opus 11 (No. 2016/21/B/ST3/ 02160) and the doctoral scholarship Etiuda 6 (No. 2018/28/ T/ST3/00154). M.E. is supported by the European Research Council project NanoThermo (ERC-2015-CoG Agreement No. 681456).

*krzysztof.ptaszynski@ifmpan.poznan.pl

[1] D. J. Evans, E. G. D. Cohen, and G. P. Morriss, Probability of Second Law Violations in Shearing Steady States, Phys. Rev. Lett. 71, 2401 (1993).
[2] G. Gallavotti and E. G. D. Cohen, Dynamical Ensembles in Nonequilibrium Statistical Mechanics, Phys. Rev. Lett. 74, 2694 (1995).

[3] J. L. Lebowitz and H. Spohn, A Gallavotti-Cohen-type symmetry in the large deviation functional for stochastic dynamics, J. Stat. Phys. 95, 333 (1999).

[4] C. Jarzynski, Microscopic analysis of Clausius-Duhem processes, J. Stat. Phys. 96, 415 (1999).

[5] U. Seifert, Entropy Production along a Stochastic Trajectory and an Integral Fluctuation Theorem, Phys. Rev. Lett. 95, 040602 (2005).

[6] M. Esposito and C. Van den Broeck, Three Detailed Fluctuation Theorems, Phys. Rev. Lett. 104, 090601 (2010).

[7] U. Seifert, Stochastic thermodynamics, fluctuation theorems and molecular machines, Rep. Prog. Phys. 75, 126001 (2012).

[8] H. Spohn, Entropy production for quantum dynamical semigroups, J. Math. Phys. (N.Y.) 19, 1227 (1978).

[9] M. Esposito, K. Lindenberg, and C. Van den Broeck, Entropy production as correlation between system and reservoir, New J. Phys. 12, 013013 (2010).

[10] G. B. Cuetara, M. Esposito, and G. Schaller, Quantum thermodynamics with degenerate eigenstate coherences, Entropy 18, 447 (2016).

[11] M. Esposito and C. Van den Broeck, Second law and Landauer principle far from equilibrium, Europhys. Lett. 95, 40004 (2011).

[12] J. M. R. Parrondo, J. M. Horowitz, and T. Sagawa, Thermodynamics of information, Nat. Phys. 11, 131 (2015).

[13] P. Strasberg, G. Schaller, T. Brandes, and M. Esposito, Quantum and Information Thermodynamics: A Unifying Framework Based on Repeated Interactions, Phys. Rev. X 7, 021003 (2017).

[14] H. Miyahara and K. Aihara, Work relations with measurement and feedback control on nonuniform temperature systems, Phys. Rev. E 98, 042138 (2018).

[15] J. Goold, M. Huber, A. Riera, L. del Rio, and P. Skrzypczyk, The role of quantum information in thermodynamicsA topical review, J. Phys. A 49, 143001 (2016).

[16] K. Maruyama, F. Nori, and V. Vedral, Colloquium: The physics of Maxwell's demon and information, Rev. Mod. Phys. 81, 1 (2009).

[17] J. C. Maxwell, The Scientific Letters and Papers of James Clerk Maxwell, Vol. 2 (Cambridge University Press, Cambridge, England, 1995).

[18] L. Szilard, Über die Entropieverminderung in einem thermodynamischen System bei Eingriffen intelligenter Wesen, Z. Phys. 53, 840 (1929) [On the decrease of entropy in a thermodynamic system by the intervention of intelligent beings, Behav. Sci. 9, 301 (1964)].

[19] S. Lloyd, Quantum-mechanical Maxwell's demon, Phys. Rev. A 56, 3374 (1997).

[20] S. W. Kim, T. Sagawa, S. De Liberato, and M. Ueda, Quantum Szilard Engine, Phys. Rev. Lett. 106, 070401 (2011).

[21] G. Schaller, C. Emary, G. Kiesslich, and T. Brandes, Probing the power of an electronic Maxwell's demon: Single-electron transistor monitored by a quantum point contact, Phys. Rev. B 84, 085418 (2011). 
[22] D. Mandal and C. Jarzynski, Work and information processing in a solvable model of Maxwell's demon, Proc. Natl. Acad. Sci. U.S.A. 109, 11641 (2012).

[23] J. J. Park, K.-H. Kim, T. Sagawa, and S. W. Kim, Heat Engine Driven by Purely Quantum Information, Phys. Rev. Lett. 111, 230402 (2013).

[24] J. V. Koski, V. F. Maisi, T. Sagawa, and J. P. Pekola, Experimental Observation of the Role of Mutual Information in the Nonequilibrium Dynamics of a Maxwell Demon, Phys. Rev. Lett. 113, 030601 (2014).

[25] J. V. Koski, A. Kutvonen, I. M. Khaymovich, T. Ala-Nissila, and J. P. Pekola, On-Chip Maxwell's Demon as an Information-Powered Refrigerator, Phys. Rev. Lett. 115, 260602 (2015).

[26] P. A. Camati, J. P. S. Peterson, T. B. Batalhão, K. Micadei, A. M. Souza, R. S. Sarthour, I. S. Oliveira, and R. M. Serra, Experimental Rectification of Entropy Production by Maxwell's Demon in a Quantum System, Phys. Rev. Lett. 117, 240502 (2016).

[27] J. V. Koski and J. P. Pekola, Maxwell's demons realized in electronic circuits, C.R. Phys. 17, 1130 (2016).

[28] K. Chida, S. Desai, K. Nishiguchi, and A. Fujiwara, Power generator driven by Maxwell's demon, Nat. Commun. 8, 15310 (2017).

[29] N. Cottet, S. Jezouin, L. Bretheau, P. Campagne-Ibarcq, Q. Ficheux, J. Anders, A. Auffèves, R. Azouit, P. Rouchon, and B. Huard, Observing a quantum Maxwell demon at work, Proc. Natl. Acad. Sci. U.S.A. 114, 7561 (2017).

[30] T. Sagawa and M. Ueda, Generalized Jarzynski Equality under Nonequilibrium Feedback Control, Phys. Rev. Lett. 104, 090602 (2010).

[31] J. M. Horowitz and S. Vaikuntanathan, Nonequilibrium detailed fluctuation theorem for repeated discrete feedback, Phys. Rev. E 82, 061120 (2010).

[32] Y. Morikuni and H. Tasaki, Quantum Jarzynski-SagawaUeda relations, J. Stat. Phys. 143, 1 (2011).

[33] D. Abreu and U. Seifert, Thermodynamics of Genuine Nonequilibrium States under Feedback Control, Phys. Rev. Lett. 108, 030601 (2012).

[34] S. Deffner and C. Jarzynski, Information Processing and the Second Law of Thermodynamics: An Inclusive, Hamiltonian Approach, Phys. Rev. X 3, 041003 (2013).

[35] T. Sagawa and M. Ueda, Role of mutual information in entropy production under information exchanges, New J. Phys. 15, 125012 (2013).

[36] P. Strasberg, G. Schaller, T. Brandes, and C. Jarzynski, Second laws for an information driven current through a spin valve, Phys. Rev. E 90, 062107 (2014).

[37] A. C. Barato and U. Seifert, Unifying Three Perspectives on Information Processing in Stochastic Thermodynamics, Phys. Rev. Lett. 112, 090601 (2014).

[38] D. Hartich, A. C. Barato, and U. Seifert, Stochastic thermodynamics of bipartite systems: Transfer entropy inequalities and a Maxwell's demon interpretation, J. Stat. Mech. (2014) P02016.

[39] J. M. Horowitz and M. Esposito, Thermodynamics with Continuous Information Flow, Phys. Rev. X 4, 031015 (2014).

[40] J. M. Horowitz and H. Sandberg, Second-law-like inequalities with information and their interpretations, New J. Phys. 16, 125007 (2014).
[41] J. M. Horowitz, Multipartite information flow for multiple Maxwell demons, J. Stat. Mech. (2015) P03006.

[42] A. Chapman and A. Miyake, How an autonomous quantum Maxwell demon can harness correlated information, Phys. Rev. E 92, 062125 (2015).

[43] P. Strasberg, G. Schaller, T. Brandes, and M. Esposito, Thermodynamics of a Physical Model Implementing a Maxwell Demon, Phys. Rev. Lett. 110, 040601 (2013).

[44] H.-P. Breuer and F. Petruccione, The Theory of Open Quantum Systems (Oxford University Press, Oxford, 2002).

[45] G. Lindblad, On the generators of quantum dynamical semigroups, Commun. Math. Phys. 48, 119 (1976).

[46] V. Gorini, A. Kossakowski, and E. C. G. Sudarshan, Completely Positive Dynamical Semigroups of N-Level Systems, J. Math. Phys. (N.Y.) 17, 821 (1976).

[47] See Supplemental Material at http://link.aps.org/ supplemental/10.1103/PhysRevLett.122.150603, which includes Refs. [10,39,44,48-56], for the derivation of the nonsecular Lindblad equation and additional discussions.

[48] G. Argentieri, F. Benatti, R. Floreanini, and M. Pezzutto, Violations of the second law of thermodynamics by a noncompletely positive dynamics, Europhys. Lett. 107, 50007 (2014).

[49] B. Palmieri, D. Abramavicius, and S. Mukamel, Lindblad equations for strongly coupled populations and coherences in photosynthetic complexes, J. Chem. Phys. 130, 204512 (2009).

[50] G. Schaller, G. Kießlich, and T. Brandes, Transport statistics of interacting double dot systems: Coherent and nonMarkovian effects, Phys. Rev. B 80, 245107 (2009).

[51] B. Wunsch, M. Braun, J. König, and D. Pfannkuche, Probing level renormalization by sequential transport through double quantum dots, Phys. Rev. B 72, 205319 (2005).

[52] J. Splettstoesser, M. Governale, and J. König, Tunnelinginduced renormalization in interacting quantum dots, Phys. Rev. B 86, 035432 (2012).

[53] M. A. Nielsen and I. L. Chuang, Quantum Computation and Quantum Information (Cambridge University Press, Cambridge, England, 2010).

[54] G. Schaller, Open Quantum Systems Far from Equilibrium (Springer, Heidelberg, 2014).

[55] M. M. Wilde, Quantum Information Theory (Cambridge University Press, Cambridge, England, 2013).

[56] G. Kiršanskas, M. Franckié, and A. Wacker, Phenomenological position and energy resolving Lindblad approach to quantum kinetics, Phys. Rev. B 97, 035432 (2018).

[57] C.-K. Chan, G.-D. Lin, S. F. Yelin, and M. D. Lukin, Quantum interference between independent reservoirs in open quantum systems, Phys. Rev. A 89, 042117 (2014).

[58] G. G. Giusteri, F. Recrosi, G. Schaller, and G. L. Celardo, Interplay of different environments in open quantum systems: Breakdown of the additive approximation, Phys. Rev. E 96, 012113 (2017).

[59] M. T. Mitchison and M. B. Plenio, Non-additive dissipation in open quantum networks out of equilibrium, New J. Phys. 20, 033005 (2018).

[60] H. M. Friedman, B. K. Agarwalla, and D. Segal, Quantum energy exchange and refrigeration: A full-counting statistics approach, New J. Phys. 20, 083026 (2018). 
[61] S. Watanabe, Information theoretical analysis of multivariate correlation, IBM J. Res. Dev. 4, 66 (1960).

[62] K. Ptaszyński, Autonomous quantum Maxwell's demon based on two exchange-coupled quantum dots, Phys. Rev. E 97, 012116 (2018).

[63] W. Rudziński and J. Barnaś, Tunnel magnetoresistance in ferromagnetic junctions: Tunneling through a single discrete level, Phys. Rev. B 64, 085318 (2001).
[64] M. Braun, J. König, and J. Martinek, Theory of transport through quantum-dot spin valves in the weak-coupling regime, Phys. Rev. B 70, 195345 (2004).

[65] N. Schuch and J. Siewert, Natural two-qubit gate for quantum computation using the XY interaction, Phys. Rev. A 67, 032301 (2003).

[66] G. Benenti, G. Casati, K. Saito, and R. S. Whitney, Fundamental aspects of steady-state conversion of heat to work at the nanoscale, Phys. Rep. 694, 1 (2017). 